\title{
Visiting two objects in the field of the ring galaxy HRG 2302 ${ }^{\star}$ (Research Note)
}

\author{
M. Faúndez-Abans ${ }^{1}$, V. P. Reshetnikov ${ }^{2}$, M. de Oliveira-Abans ${ }^{1,3}$, A. C. Krabbe ${ }^{4}$, \\ P. C. da Rocha-Poppe ${ }^{5,6}$, V. A. Fernandes-Martin ${ }^{5,6}$, E. B. Amôres ${ }^{5,6}$, and P. Freitas-Lemes ${ }^{4}$ \\ ${ }^{1}$ MCTI/Laboratório Nacional de Astrofísica, Rua Estados Unidos 154, CEP 37504-364, Itajubá, MG, Brazil \\ 2 St.Petersburg State University, Universitetsky pr. 28, 198504 St.Petersburg, Russia \\ 3 UNIFEI, Instituto de Engenharia de Produção e Gestão, Av. BPS, 1303, Pinheirinho, CEP 37500-903, Itajubá, MG, Brazil \\ ${ }^{4}$ Universidade do Vale do Paraíba, Av. Shishima Hifumi 2911, 12244-000 São José dos Campos, SP, Brazil \\ e-mail: angela.krabbe@gmail.com; priscila@univap.br \\ UEFS, Departamento de Física, Av. Transnordenstina, S/N, Novo Horizonte, CEP 44036-900, Feira de Santana, BA, Brazil \\ ${ }^{6}$ UEFS, Observatório Astronômico Antares, Rua da Barra, 925, Jardim Cruzeiro, CEP 44015-430, Feira de Santana, BA, Brazil \\ e-mail: [paulopoppe;vmartin1963]@gmail.com; ebamores@uefs.br
} e-mail: [max;mabans]@lna.br e-mail: v.reshetnikov@spbu.ru

Received 27 October 2014 / Accepted 9 December 2014

\section{ABSTRACT}

\begin{abstract}
Aims. We investigate the nature of two galaxies that are located in the field of the ring galaxy HRG 2302.
Methods. This study is based on direct BVRI imaging and long-slit spectrophotometric data in the range of 4000-9500 $\AA$ obtained with the $1.6 \mathrm{~m}$ telescope of the Observatório do Pico dos Dias, Brazil. The spectra were used to determine the radial velocity.

Results. The primary objective of the retrieval of the photometric data was to identify the fine structures of objects $\mathrm{H}$ and I. In addition, we performed image processing and made a photometric analysis to obtain the integrated standard BVRI magnitudes. The contour maps show evidence of material connecting both galaxies, suggesting that they might be interacting close companions. We estimated redshifts of $z=0.0689$ and $z=0.0692$. The spectra of the two galaxies resemble those of an early-type galaxy. The fact that the objects have a small radial-velocity difference and the structures around object I suggest an ongoing tidal interaction between the two galaxies.

Conclusions. The H-I system seems to be composed of two early-type spiral galaxies ( $\mathrm{S} 0 / \mathrm{Sa}$ ). Galaxy I shows evidence of tidal perturbation: an off-centered bulge, some material extending along the NE direction, and structures that have been enhanced by image filtering procedures. There are some dwarf objects around it. Neither object shows evidence of nuclear activity.
\end{abstract}

Key words. galaxies: general - galaxies: interactions - galaxies: peculiar

\section{Introduction}

Galaxies have been categorized according to their shape. Historically, peculiar galaxies have also been classified in this way (e.g., Vorontsov-Velyaminov 1959, 1974; Arp 1966; Arp \& Madore 1987; Whitmore et al. 1990; Faúndez-Abans \& de Oliveira-Abans 1998a; Gnedin 2009; Moiseev et al. 2011). This feature is, nonetheless, not sufficient to understand the properties and structure formation of these objects. To contribute to this discussion, da Rocha-Poppe et al. (in prep.) have begun a long-term observational program based on a sample of galaxies in the range $0.02<z<0.07$ selected from the Arp-Madore catalog (Arp \& Madore 1987). In the fields of these peculiar galaxies, some other galaxies are located that also show peculiarities such as tidal interaction, apparent perturbations, grouping in pairs and triplets, alignment in well-defined chains such as FOA 00103, and arc-like galaxies such as HRG 2302.

* Based on observations carried out at the Observatório do Pico dos Dias (OPD), which is operated by LNA/MCTI, and public data from the LNA database.
In the course of this project, some faint field objects have caught our attention, which are worth observing. In the field of HRG 2302 ( $z \approx 0.020$; Myrrha et al. 1999 - hereafter MVFOS; see also NASA/IPAC - Extragalactic Database - NED) there are two such objects, named $\mathrm{H}$ and $\mathrm{I}$, which lie angularly close to HRG 2302. We refer to Fig. 1 of MVFOS for a view of the field and names of many of the other field objects.

In this note, we present preliminary results of complementary direct imagery and recent spectroscopic observations of the objects $\mathrm{H}$ and I, a probable interacting pair of galaxies.

\section{Observations and data reduction}

We retrieved public photometric MVFOS data on HRG 2302 from the OPD/LNA data-bank. The photometric observations were performed on the $1.6 \mathrm{~m}$ telescope of the Observatorio do Pico dos Dias, Brazil, equipped with direct imaging camera $^{1} \# 1$ and CCD \#048 (EEV CCD-05-20-0-202, thick, frontilluminated, UV-coated, $770 \times 1152$ square pixels, $22.5 \mu \mathrm{m}$ each, readout noise $=11.55 e^{-} \mathrm{ADU}^{-1}$ and gain $=10.05 e^{-} \mathrm{ADU}^{-1}$ ), and BVRI filters (as defined in Bessel 1990). The plate scale

1 www.lna.br/instrum/camara/camara.html 
Table 1. Basic properties of $\mathrm{H}$ and $\mathrm{I}$.

\begin{tabular}{lccl}
\hline \hline Parameter & Object H & Object I & Ref. \\
\hline RA (2000) & $16^{\mathrm{h}} 32^{\mathrm{m}} 37^{\mathrm{s}} .3$ & $16^{\mathrm{h}} 32^{\mathrm{m}} 41^{\mathrm{s}} .3$ & MVFOS \\
Dec (2000) & $-81^{\circ} 06^{\prime} 47^{\circ} .7$ & $-81^{\circ} 06^{\prime} 36.6$ & MVFOS \\
$l$ & 310.797 & 310.801 & this work \\
$b$ & -21.854 & -21.855 & this work \\
Morph. type & $\mathrm{S} 0 / \mathrm{Sa}$ & $\mathrm{Sa}$ & this work \\
$z$ & 0.0689 & 0.0692 & this work \\
$B$ & $19.40 \pm 0.25$ & $19.75 \pm 0.25$ & this work \\
$V$ & $18.70 \pm 0.95$ & $18.29 \pm 0.95$ & this work \\
$R$ & $18.03 \pm 0.80$ & $17.59 \pm 0.80$ & this work \\
$I$ & $17.55 \pm 0.25$ & $16.80 \pm 0.25$ & this work \\
$M_{V}^{0}$ & -19.2 & -19.7 & this work \\
Diameter & $13 \mathrm{kpc}$ & $16 \mathrm{kpc}$ & this work \\
$B / T(V$ filter $)$ & 0.6 & 0.3 & this work \\
\hline
\end{tabular}

was $0.30 \mathrm{pix}^{-1}$. The photometric analysis presented here is based on a set of 12 CCD images obtained under seeing $<2$ ". The image processing and data analysis were made using standard techniques and IRAF and STSDAS packages.

We performed the spectroscopic observations on the same telescope, with a Cassegrain spectrograph ${ }^{2}$ and CCD \#106 (SITe SI003AB, thin, back-illuminated, $1024 \times 1024$ square pixels, $24 \mu \mathrm{m}$ each, readout noise of $4.1 e^{-}$and gain of $\left.5.0 e^{-} \mathrm{ADU}^{-1}\right)$. The 3 arcsec slit was positioned to encompass the whole $\mathrm{H}$ edgeon "disk" plus the bulge of I, and centered on H. The 300line $\mathrm{mm}^{-1}$ grating was centered at $650 \mathrm{~nm}$, with a dispersion of $0.24 \mathrm{~nm} \mathrm{~mm}^{-1}$ and resolution of 2.8 pixels (FWHM). Two spectra of $2400 \mathrm{~s}$ each were obtained. The stars used for extinction and flux calibration are tertiary standards from Baldwin \& Stone (1984), as revised by Hamuy et al. (1992; see also Hamuy et al. 1994).

We cross-correlated our observed spectra with three galaxies and star templates with good signal-to-noise ratio. These results were compared with the composite absorption-line template "fabtemp97" distributed by the RVSAO/IRAF external package. We adopted the redshift value from the best highest correlated coefficient template.

\section{Results and discussion}

\subsection{Image enhancement}

The first goal of retrieving the photometric data was to study the fine morphological structures of objects $\mathrm{H}$ and I. The image processing and photometric analysis were made following the same procedure as in MVFOS. Our integrated BVRI magnitudes as well as the absolute magnitudes corrected for the Milky Way absorption (Schlafly \& Finkbeiner 2011) and $k$-correction (Chilingarian et al. 2010) are displayed in Table 1 and match previous determinations as listed by MVFOS. Galaxy I is redder than galaxy $\mathrm{H}$. The residual $B$-filter image in Fig. 1 suggests that object I may be a face-on spiral or an elliptical, and $\mathrm{H}$ seems to be an edge-on disk galaxy. Some of the image enhancement tools employed by Faúndez-Abans \& de Oliveira-Abans (1998b) were used in this work to search for fine structures in and around both galaxies. Figure 1 reveals that object G (MFVOS) is probably composed of two objects, hereafter named G1 and G2. This image suggests an overall blue stellar component and extended material not only between them (to the north of $\mathrm{H}$ ), but also to the NE of object I (tidal tail? rim?).

\footnotetext{
2 www.lna.br/instrum/cassegr/cassegr.html
}

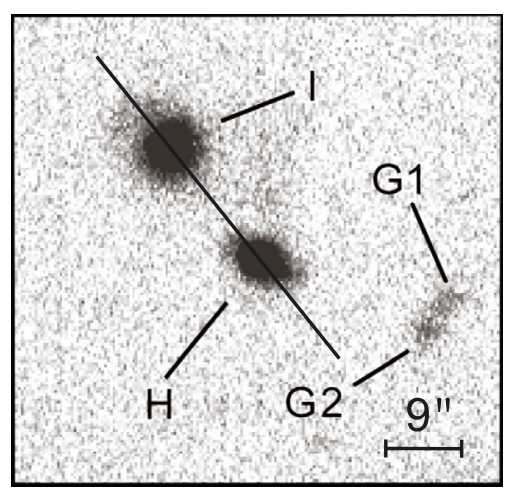

Fig. 1. Trimmed residual $B$ image after low-pass filtering. Two new objects are enhanced: $\mathrm{G} 1$ and $\mathrm{G} 2$. The $\mathrm{PA}=40^{\circ}$ slit position is displayed. North is up, east is on the left.

Figure 2 displays contour maps in the $R$-band (left) and in white light (right); these maps confirm the extended material between $\mathrm{H}$ an I. They also suggest that G1 and G2 share a common envelope. Both scenarios might be explained by ongoing interactions between $\mathrm{H}$ and I and between objects G1 and G2. The left panel also shows that there is additional extended material on the eastern side of galaxy I, and that G2 may be slightly redder than G1. Even though both $\mathrm{H}$ and I bulges are round, one sees that (i) the galaxy halo of I is elliptical-like; (ii) its major axis lies almost perpendicular to the minor axis of the $\mathrm{H}$ galaxy; (iii) the external $R$-band isophotes of $\mathrm{H}$ are boxy; (iv) the luminous nuclei do not lie on the geometrical centers of their respective bulge isophotes, but are slightly displaced one toward the other. This behavior can also be seen in peculiar ring galaxies of Solitaire type in early stages of interaction (see Wenderoth et al. 2011) and ongoing tidal interaction (see Faúndez-Abans et al. 2013).

By performing two-dimensional fitting with the GALFIT package (Peng et al. 2010) on the images, we found that the bulges on both galaxies contribute significantly to the total luminosity (see last line of Table 1 for bulge-to-total ratio in the $V$ passband). Therefore the galaxies can be classified as earlytype spirals.

We have tentatively produced a Fourier hologram of the $R$ frame (Fig. 3) to enhance the fine morphological structures in both objects (see Faúndez-Abans \& de Oliveira-Abans 1998b). As a byproduct, a number of small objects in the field have also been highlighted; they have been named k1-k6 knots and the S-object. Except for k1 and k4, they seem to be associated with the I galaxy. Should future work prove that the extended material of the $\mathrm{N}$ of galaxy $\mathrm{H}$ is a bridge to galaxy $\mathrm{I}$, then maybe $\mathrm{S}$ and $\mathrm{k} 3$ are part of it. We were unable to perform a more detailed photometric analysis at this stage because of the low signal of the images. Table 2 displays the coordinates of G1 and G2.

\subsection{Spectroscopy}

Figure 4 shows the spectra that were obtained with the slit aligned to cover galaxies $\mathrm{H}$ and I (both targets far south with an airmass around 2.0). As can be seen, they are rather noisy. Overplotted in both panels is the identification of the main absorption features: $\mathrm{G}$ band $\lambda 4305 \AA, \mathrm{H} \beta, \operatorname{MgIb} \lambda 5174 \AA$, $\mathrm{MgH} \lambda 5269 \AA$, NaID $\lambda 5892 \AA$, and the TiO band in $\lambda 6250$. The signal of the $\mathrm{H}$ galaxy was very poor. The radial velocity for each galaxy was estimated by the cross-correlation technique. Using IRAF/RVXCOR, we cross-correlated our observed spectra with three galaxies and star templates with high signal-to-noise ratio. 

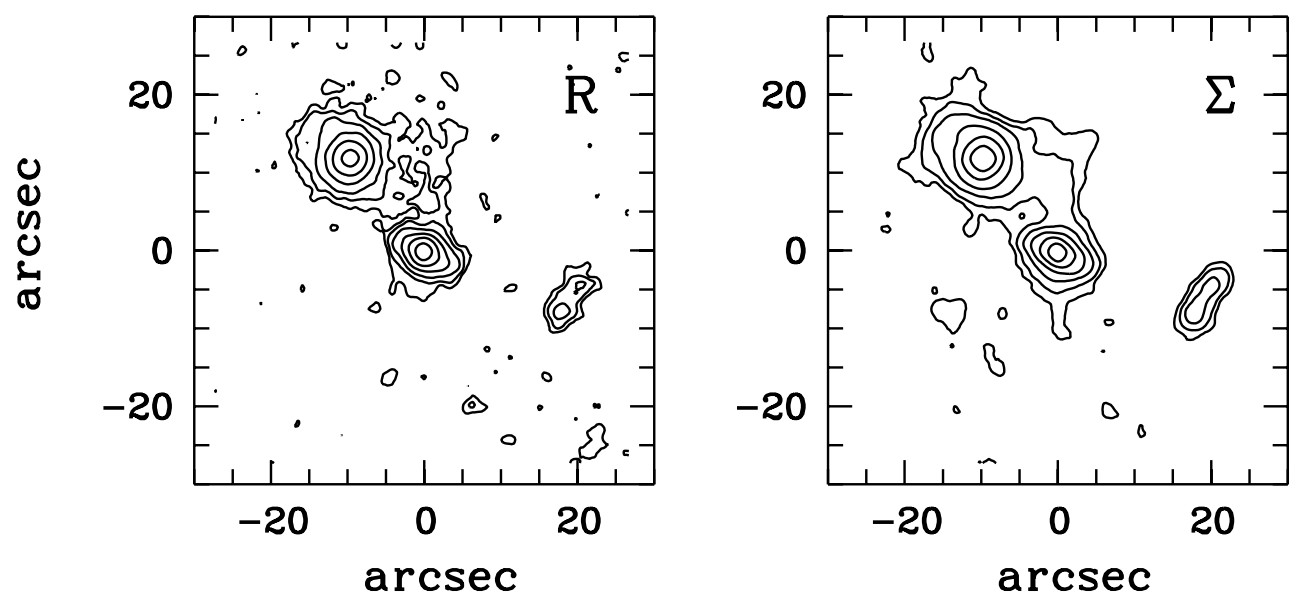

Fig. 2. Contour maps for the $R$ filter (left panel) and for the sum-of-all-filter images (right panel). Both axes are in arcseconds.

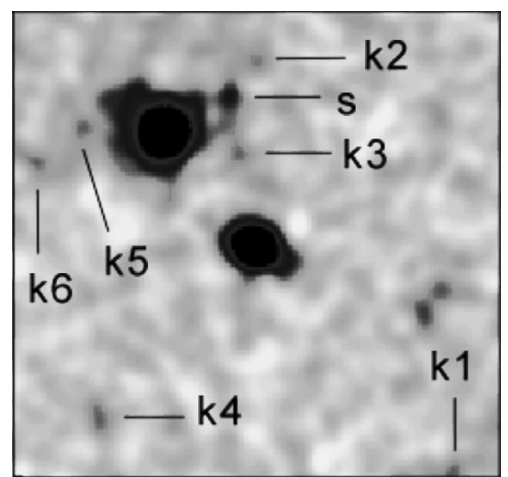

Fig. 3. Fourier hologram in the $R$-band enhancing faint structures in the two objects and the field. The individualization of objects G1 and G2 is clear.

These results were checked with the composite absorption-line template "fabtemp97" distributed by the $\mathrm{RVSAO}^{3} / \mathrm{IRAF}$ external package. We adopted the redshift value from the best highly correlated coefficient template. The spectra had significant fringing in the red, from $7500 \AA$ to $9500 \AA$, therefore it was not used in our analysis. No emission lines were detected along the entire slit in either objects. The derived heliocentric velocity for $\mathrm{H}$ is $20650 \pm 449 \mathrm{~km} \mathrm{~s}^{-1}(z=0.0688)$, and for I it is $20746 \pm 106 \mathrm{~km} \mathrm{~s}^{-1}(z=0.0692)$.

Based on these spectroscopic results, the projected separation of $r_{\mathrm{p}} \sim 20 h^{-1} \mathrm{kpc}$ and the radial velocity difference of $\Delta V \sim 96 \mathrm{~km} \mathrm{~s}^{-1}$, we confirm that the two galaxies are an interacting pair. These values agree well with some recent statistical work on the construction of galaxy pair catalogs, see Mesa et al. (2014) and reference therein. In this recent paper, the authors discuss interacting galaxy pairs with evidence of tidal features in the Sloan Digital Sky Survey data release 7 (SDSS-DR7) for $z<0.1$ with $r_{\mathrm{p}}<50 h^{-1} \mathrm{kpc}$ and $\Delta V<500 \mathrm{~km} \mathrm{~s}^{-1}$.

As described earlier, galaxy I shows evidence of tidal interaction perturbation, but no evidence of nuclear activity (Fig. 4, top panel). This is an interesting result because Lambas et al. (2003) found that galaxies with a close companion within $<25 h^{-1} \mathrm{kpc}$ and $\Delta V<100 \mathrm{~km} \mathrm{~s}^{-1}$ show a higher star formation

\footnotetext{
3 The RVSAO IRAF (Radial Velocity Package for IRAF) external package was developed at the Smithsonian Astrophysical Observatory. Full documentation of this software, including numerous examples of its use, is online at http://tdcwww. harvard. edu/iraf/rvsao/.
}

Table 2. Positions for objects G1 and G2.

\begin{tabular}{lcc}
\hline \hline Parameter & Object G1 & Object G2 \\
\hline RA (2000) & $16^{\mathrm{h}} 32^{\mathrm{m}} 29^{\mathrm{s}} .07$ & $16^{\mathrm{h}} 32^{\mathrm{m}} 29^{\mathrm{s}} .36$ \\
Dec (2000) & $-81^{\circ} 06^{\prime} 51^{\mathrm{s}} .34$ & $-81^{\circ} 06^{\prime} 54.16$ \\
$l$ & 310.792 & 310.792 \\
$b$ & -21.850 & -21.851 \\
\hline
\end{tabular}

activity than isolated galaxies with similar redshift and luminosity distributuion.

To compute distance-dependent quantities, we adopted the mean redshift of the binary system as 0.069 . Assuming a standard flat cosmology, we obtained the luminosity distance of $310.9 \mathrm{Mpc}$ and the scale $1.32 \mathrm{kpc} /{ }^{\prime \prime}$. The projected angular distance between $\mathrm{H}$ and I galaxies is therefore $15^{\prime \prime} 3$ or $20.2 \mathrm{kpc}$, and galaxies $\mathrm{H}+\mathrm{I}$ form a close interacting system.

\subsection{Stellar population synthesis}

We used the stellar population synthesis code STARLIGHT (Cid Fernandes et al. 2004) to describe the age distributions and metallicities of the stellar populations that fit the integrated light spectrum of object I. For object $\mathrm{H}$ it is not possible to perform the stellar population synthesis because of the low signal-tonoise ratio of the spectrum. This code is extensively discussed in Cid Fernandes et al. $(2004,2005)$, and it is built upon computational techniques that originally were developed for empirical population synthesis with additional ingredients from evolutionary synthesis models. This method was used by Krabbe et al. (2011) and Faúndez-Abans et al. $(2012,2013)$ and has been successful in describing the stellar population in interacting galaxies. Briefly, the code fits an observed spectrum with a combination of N single stellar populations (SSPs) from the models of Bruzual \& Charlot (2003). These models are based on a highresolution library of observed stellar spectra, which allows for detailed spectral evolution of the SSPs across the wavelength range of 3200-9500 $\AA$ with a wide range of metallicities. We used the Padova 1994 tracks, as recommended by Bruzual \& Charlot (2003), with the Salpeter initial mass function (Salpeter 1955). Extinction is modeled by STARLIGHT as due to foreground dust, using the Large Magellanic Cloud average reddening law of Gordon et al. (2003) with $R_{V}=3.1$, and parametrized by the $V$-band extinction $A_{V}$. The SSPs used in this work cover 15 ages, $t=0.001,0.003,0.005,0.01,0.025,0.04,0.1,0.3$, 

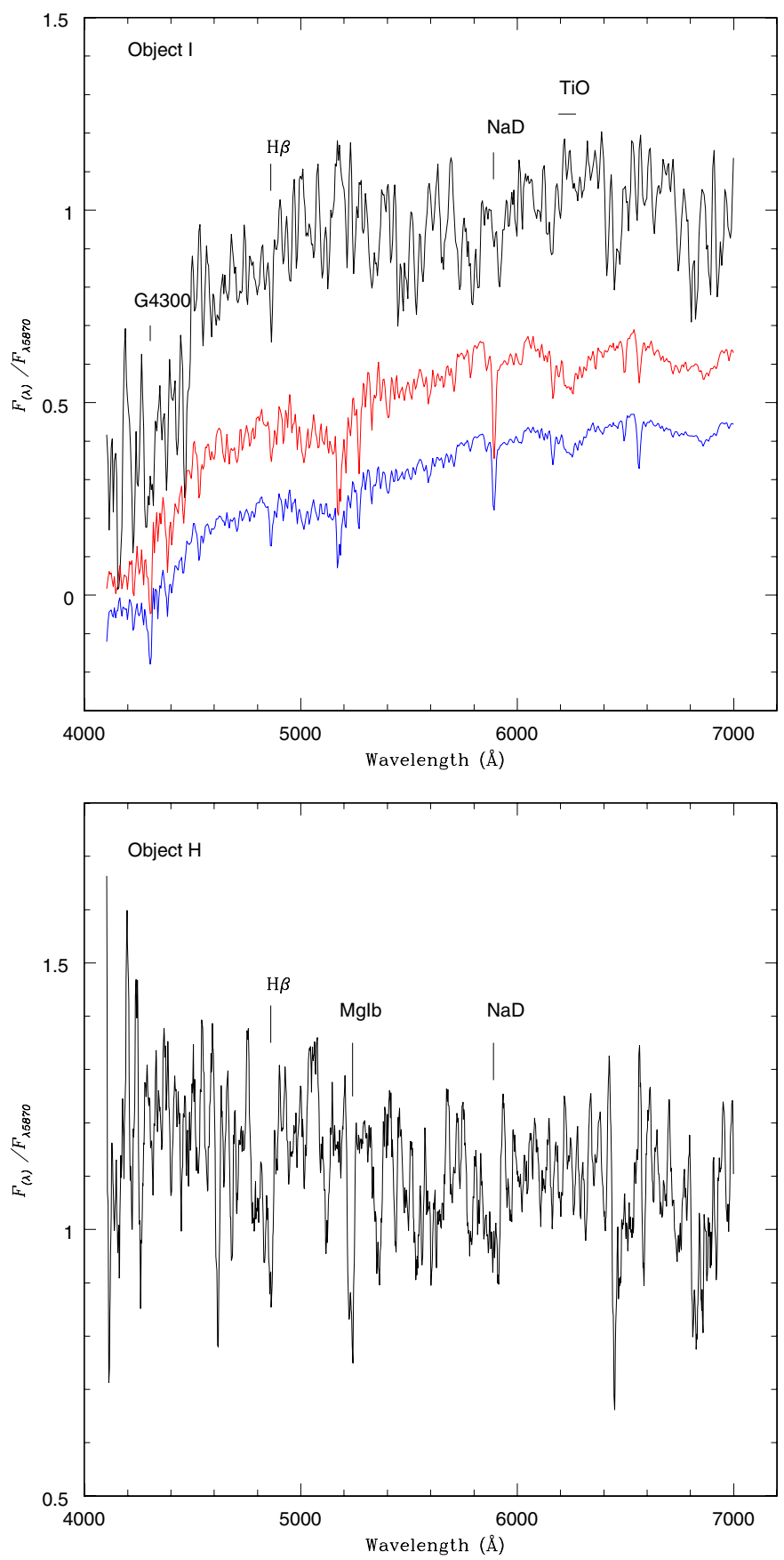

Fig. 4. Spectra of the two galaxies. Upper panel: observed spectrum of object I corrected for reddening and the shifted synthesized spectra fitted considering three metallicities (red) and with a fixed metallicity (blue). Lower panel: observed spectrum of object $\mathrm{H}$. The identification of the main absorption features is overplotted in both panels.

$0.6,0.9,1.4,2.5,5,11$, and $13 \mathrm{Gyr}$, and three metallicities, $Z=0.2 Z_{\odot}, 1 Z_{\odot}$, and $2.5 Z_{\odot}$, adding to $45 \mathrm{SSP}$ components. The fitting is carried out using a simulated annealing plus Metropolis scheme, with regions around emission lines and bad pixels excluded from the analysis. The upper panel of Fig. 4 shows the observed spectrum corrected for reddening and the model stellar population integrated light spectrum of object I. The synthesized spectrum fits the observed one closely. The results indicate that this galaxy is an old object, with $100 \%$ of the flux contribution at $\lambda 5870 \AA$ being provided by a stellar population of $13 \mathrm{Gyr}$, with $Z=2.5 Z_{\odot}$ and $A_{V}=0.36$. This spectrum is dominated principally by the bulge signal and does not represent the whole galaxy; the age estimate then is valid only for its bulge.

It is important to emphasize that the synthesis methods suffer from the well-known degeneracy problem, that is, the nonuniqueness of the best-fit solution, which in our case can be significant because the spectrum has few absorption lines. In addition, the fit is mostly being made by the shape over a wide wavelength range, which is most susceptible to errors in the extinction and/or reddening corrections. The blue spectrum of the upper panel of Fig. 4 shows a model stellar population obtained with a fixed metallicity at $Z=0.2 Z_{\odot}$. Likewise, this synthesized spectrum fits the observed one closely and results in an old object, but with $A_{V}=0.63$, slightly higher than the previous one.

\section{Concluding remarks}

We reported observational results for galaxies $\mathrm{H}$ and I, which are both in the field of the ring galaxy HRG 2302. In summary, our main results are the following:

- The H-I system seems to be composed of an S0-Sa and an Sa galaxy (see Table 1). Integrated colors indicate that galaxy $\mathrm{H}$ is bluer than galaxy I.

- The I galaxy shows evidence of tidal perturbations such as an off-centered bulge and a probable tidal tail in the NE direction. Some morphological structures have been enhanced by image filtering procedures.

- The contour maps show evidence of extended material connecting both galaxies (bridge?), suggesting that they might be close interacting companions.

- The small velocity difference reinforces the conclusion that they are a bound system.

- Neither object shows any evidence of nuclear activity.

- The stellar population synthesis results indicate that the bulge of galaxy I is an old object, with a stellar population of 13 Gyr.

We are preparing an observational program with a larger telescope to study galaxies G1 and G2 in detail.

Acknowledgements. This work was partially supported by the Ministério da Ciência, Tecnologia e Inovação (MCTI), Laboratório Nacional de Astrofísica (LNA), St. Petersburg State University research grant 6.38.71.2012, and Universidade do Vale do Paraíba - UNIVAP. A.C.K. acknowledges FAPESP support process 2010/01490-3. This publication makes use of data products from the Two Micron All Sky Survey, which is a joint project of the University of Massachusetts and the Infrared Processing and Analysis Center/California Institute of Technology, funded by the National Aeronautics and Space Administration and the National Science Foundation. This research made use of the NASA/ IPAC Infrared Science Archive, which is operated by the Jet Propulsion Laboratory, California Institute of Technology, under contract with the National Aeronautics and Space Administration.

\section{References}

Arp, H. 1966, ApJS, 14, 1

Arp, H. C., \& Madore, B. F. 1987, Catalogue of Southern Peculiar Galaxies and Associations (Toronto: Clarke-Irwin)

Baldwin, J. A., \& Stone, R. P. S. 1984, MNRAS, 206, 241

Bessel, M. 1990, PASP, 102, 1181

Bruzual, G., \& Charlot, S. 2003, MNRAS, 344, 1000

Chilingarian, I. V., Melchior A.-L., \& Zolotukhin, I. Yu. 2010, MNRAS, 405 1409

Cid Fernandes, R., Gu, Q., Melnick, J., et al. 2004, MNRAS, 355, 273 
M. Faúndez-Abans et al.: Visiting two objects in the field of the ring galaxy HRG 2302 (RN)

Cid Fernandes, R., Mateus, A., Sodré, L., Stasińska, G., \& Gomes, J. M. 2005, MNRAS, 358, 363

Faúndez-Abans, M., \& de Oliveira-Abans, M. 1998a, A\&A, 129, 357

Faúndez-Abans, M., \& de Oliveira-Abans, M. 1998b, A\&A, 128, 289

Faúndez-Abans, M., Krabbe, A.C, de Oliveira-Abans, M., et al. 2012, A\&A, 543, A64

Faúndez-Abans, M., de Oliveira-Abans, M., Krabbe, A. C., et al. 2013, A\&A, 558, A 13

Gnedin, N. Y. 2009, Nature, 461, 43

Gordon, K. D., Clayton, G. C., Misselt, K. A., et al. 2003, ApJ, 594, 279 Hamuy, M., Walker, A. R., Suntzeff, N. B., et al. 1992, PASP, 104, 533

Hamuy, M., Suntzeff, N. B., Heathcote, S. R., et al. 1994, PASP, 106, 566

Krabbe, A. C., Pastoriza, M. G., Winge, C., et al. 2011, MNRAS, 416, 38

Lambas, D. G., Tissera, P. B., Alonso, M. S., \& Coldwell, G. 2003, MNRAS, 346,1189
Mesa, V., Duplacic, F., Alonso, S., et al. 2014, MNRAS, 438, 1784

Moiseev, A. V., Smirnova, K. I., Smirnova, A. A., \& Reshetnikov, V. P. 2011, MNRAS, 418, 244

Myrrha, M. L. M., Vaz, L. P. R., Faúndez-Abans, M., et al. 1999, A\&A, 351, 860

Peng, Ch.Y., Impey, Ch.D., \& Rix, H.-W. 2010, AJ, 139, 2097

Salpeter, E. E. 1955, ApJ, 121, 161

Schlafly, E. F., \& Finkbeiner, D. P. 2011, ApJ, 737, 103

Vorontsov-Velyaminov, B. A. 1959, Atlas and catalog of interacting galaxies, Stenberg Institute, Moscow State University

Vorontsov-Velyaminov, B. A. 1974, The Observatory, 94, 319

Wenderoth, E., Faúndez-Abans, M., Krabbe, A. C., et al. 2011, A\&A, 529, A157

Whitmore, B. C., Lucas, R. A., McElroy, D. B., et al. 1990, AJ, 100, 1489 